\title{
On tensor products Gorenstein rings
}

\author{
By \\ Kei-ichi Watanabe, Takeshi Ishikawa, Sadao Tachibana \\ and Kayo Otsuka
}

(Received July 10, 1969)

\section{Introduction}

Among local rings there is a hierarchy: regular $\Rightarrow$ complete intersection $\Rightarrow$ Gorenstein $\Rightarrow$ Macaulay. These concepts are extended to non-local rings; for example a ring $A$ is regular if for all prime ideal $\mathfrak{p}$ of $A, A_{\mathfrak{p}}$ is a regular local ring.

In this paper, we shall investigate if these properties are conserved under tensor product operations. It is well known that the tensor product $B \otimes_{A} C$ of regular rings are not regular in general, even if we assume $A, B$ and $C$ are fields.

But it can be shown that under a suitable condition tensor products of regular rings are complete intersections. For Macaulay rings and Gorenstein rings, it is proved implicitly in [2], that tensor product $B \otimes_{A} C$ of Macaulay rings are again Macaulay if we assume $B$ is $A$ flat and $C$ is finitely generated over $A$, and we shall show that the same is true for Gorenstein rings.

Part I, which is the main part of the present paper, was written by the first author. If one assume that $A$ is a field, then the treatment is much simpler and we have a similar result under a weaker finiteness condition. The case was given by the second and the third authors and is the contents of Part II. On the other hand, the case of complete tensor products over a field was observed by the last author, who wrote Part III. 
These three groups of authors obtained results rather independently. But, because of the close relationship among these results, the authors put their manuscripts together and obtained the present paper.

\section{Part I}

\section{Notations and quoted theorems}

A ring means always commutative Noetherian ring with unit. A prescheme is always locally Noetherian prescheme.

(1.1) Definition. A local ring $A$ is a complete intersection if there exist a regular local ring $B$ and $B$-sequence $\left(x_{1}, \cdots, x_{d}\right)$ such that $A \cong B /\left(x_{1}, \cdots, x_{d}\right)$.

(1.2) Definition. A local ring $A$ is an S-complete intersection if its completion $\hat{A}$ is a complete intersection in the sense of (1.1).

(1.3) Proposition. (Scheja [5]) If $A$ is a homomorphic image of a regular local ring, then $A$ is a complete intersection $\Leftrightarrow A$ is an S-complete intersection.

Henceforth, we shall use the word "complete intersection" for the sense of $S$-complete intersection and write C.I. for short.

(1.4) Proposition. (Scheja [5]) If $A$ is a local ring and ( $x_{1}$, $\left.\cdots, x_{d}\right)$ is an A-sequence, then:

$A$ is a C.I. $\Leftrightarrow A /\left(x_{1}, \cdots, x_{d}\right)$ is a C.I.

The definitions and properties of Macaulay rings and Gorenstein rings are found respectively in [6] and [2]. We do not refer to them, now.

(1.5) Definition. A prescheme $\left(X, \mathcal{O}_{X}\right)$ is regular (resp. C.I., Gorenstein, Macaulay) if for every $x \in X$, the local ring $\mathcal{O}_{x}$ is regular (resp. C.I., Gorenstein, Macaulay).

(1.6) Definition. A ring $A$ is regular (resp. C.I., Gorenstein, Macaulay) if $\operatorname{Spec}(A)$ is regular (resp. C.I., Gorenstein, Macaulay). 
(1.7) Definition. A morphism of preschemes $f: X \rightarrow Y$ is Gorenstein (resp. Macaulay, C.I.) if $f$ is flat and if each fibre $f^{-1}(y)$ $=X \times_{\mathrm{Y}} \operatorname{Spec}(k(y))$ is Gorenstein (resp. Macaulay, C.I.).

\section{The theorems and proofs}

Theorem 1. Let $(A, \mathfrak{m})$ and $(B, \mathfrak{n})$ be local rings, $f: A \rightarrow B$ a local homomorphism, making $B$ a flat A-module. Then:

(1) $B$ is Macaulay $\Leftrightarrow A$ and $B / \mathfrak{m} B$ are Macaulay.

(2) $B$ is Gorenstein $\Leftrightarrow A$ and $B / \mathrm{m} B$ are Gorenstein.

(3) $A$ is regular and $B / \mathrm{nt} B$ is a C.I. $\Leftrightarrow B$ is a C.I.

Theorem $\mathbf{1}^{\prime}$. If $f: X \rightarrow Y$ is a flat and surjective morphism of preschemes, then:

$(1)^{\prime} X$ is Macaulay $\Leftrightarrow Y$ and $f$ are Macaulay.

$(2)^{\prime} X$ is Gorenstein $\Leftrightarrow Y$ and $f$ are Gorenstein.

(3)' If $Y$ is regular and $f$ is C.I., then $X$ is C.I.

(The condition " $f$ is surjective" is necessary only to deduce that $Y$ is Macaulay (resp. Gorenstein) from $X$ is Macaulay (resp. Gorenstein) in $(1)^{\prime}$ and $\left.(2)^{\prime}\right)$.

Proof. (1) is in [2] (VI. 6.3.5.).

(3) As $A$ is regular, $m$ is generated by an $A$-sequence $\left(x_{1}, \cdots, x_{d}\right)$ $(d=\operatorname{dim} A)$. As $f$ is flat, $\left(x_{1}, \cdots, x_{d}\right)$ is a $B$-sequence. By (1.3), $B$ is a C.I. $\Leftrightarrow B / \mathrm{mt} B$ is a C.I.

(2) We prove the assertion by induction on $d=\operatorname{dim}(B / \mathfrak{m} B)$. By (1), we may assume $A, B$ and $B / \mathrm{m} B$ are Macaulay.

Case 1. $d=0$.

Let $\mathfrak{q}$ be an ideal of $A$, generated by a parameter system. As $A$ is Macaulay, $\mathfrak{q}$ is generated by an $A$-sequence. As $B$ is $A$-flat, an $A$-sequence is also a $B$-sequence. So, $A$ is Gorenstein $\Leftrightarrow A / \mathfrak{q}$ is Gorenstein, and $B$ is Gorenstein $\Leftrightarrow B / \mathfrak{q} B$ is Gorenstein.

Thus we may assume $\operatorname{dim} A=0$ and $\operatorname{dim} B=0 . \quad$ As $\operatorname{dim} A=0, A$ is Gorenstein $\Leftrightarrow 0: \mathrm{m} \cong A / \mathrm{m} \Leftrightarrow \operatorname{Hom}_{A}(A / \mathrm{m}, A) \cong A / \mathrm{m}$. Now, let us 
assume $A$ and $B / \mathrm{m} B$ are Gorenstein. Then:

$$
\begin{aligned}
\operatorname{Hom}_{B}(B / \mathfrak{n}, B) & \cong \operatorname{Hom}_{B}\left(B / \mathfrak{n}, \operatorname{Hom}_{B}(B / \mathfrak{m} B, B)\right) \\
& \cong \operatorname{Hom}_{B}\left(B / \mathfrak{n}, \operatorname{Hom}_{A}(A / \mathfrak{m}, A) \otimes_{A} B\right) \\
& \cong \operatorname{Hom}_{B}\left(B / \mathfrak{n}, A / \mathfrak{m} \otimes_{A} B\right) \\
& \cong \operatorname{Hom}_{B}(B / \mathfrak{n}, B / \mathfrak{m} B) \cong B / \mathfrak{n} .
\end{aligned}
$$

Thus $B$ is Gorenstein. Conversely, let us assume $A$ or $B / \mathrm{m} B$ is not Gorenstein. Then $\operatorname{Hom}_{B / \mathfrak{m} B}(B / \mathfrak{n}, b / \mathfrak{m} B) \cong(B / \mathfrak{n})^{p}, \operatorname{Hom}_{A}(A / \mathfrak{m}, A)$ $\cong(A / \mathfrak{m})^{q}, p q>1$. Then by the same argument as above, $\operatorname{Hom}_{B}(B / \mathfrak{n}, B)$ $\cong(B / \mathfrak{n})^{p q}$ and $B$ is not Gorenstein.

Case 2. $d>0$.

As $B / \mathfrak{m} B$ is Macaulay and $\operatorname{dim}(B / \mathfrak{m} B)>0$, we can find an element $x \in B$ such that $x$ is not a zero divisor in $B / \mathrm{m} B$. Then by [2] ( $\left(0_{\mathrm{III}}\right.$ 10.2. 4.) $x$ is not a zero divisor in $B$ and $\bar{B}=B / x B$ is $A$ flat. By the induction hypothesis, $\bar{B}$ is Gorenstein $\Leftrightarrow A$ and $\bar{B} / \mathrm{m} \bar{B}$ are Gorenstein. But as $\bar{B} / \mathrm{m} \bar{B} \cong(B / \mathrm{m} B) / x(B / \mathrm{m} B), \bar{B} / \mathrm{m} \bar{B}$ is Gorenstein $\Leftrightarrow B / \mathfrak{m} B$ is Gorenstein. Thus $B$ is Gorenstein $\Leftrightarrow \bar{B}$ is Gorenstein $\Leftrightarrow A$ and $\bar{B} / \mathrm{m} \bar{B}$ are Gorenstein. $\Leftrightarrow A$ and $B / \mathrm{m} B$ are Gorenstein, and we are done.

Remark. It is clear that in the proof of (3), the following is included:

(4) $A$ is regular and $B$ is a C.I. $\Rightarrow B / \mathrm{m} B$ is a C.I.

Corollary 1. If $A$ is a Gorenstein ring (resp. is a C.I.), so is $A[X]$.

Proof. The natural map $\operatorname{Spec}(A[X]) \rightarrow \operatorname{Spec}(A)$ is clearly Gorenstein. As for the C.I. property, we may assume $A$ is local, $A \cong B /\left(x_{1}, \cdots, x_{d}\right), B$ is regular and $\left(x_{1}, \cdots, x_{d}\right)$ a $B$-sequence. Then $A[X] \cong B[X] /\left(x_{1}, \cdots, x_{d}\right) B[X], B[X]$ is regular and $\left(x_{1}, \cdots, x_{d}\right)$ a $B[X]$-sequence. So $A[X]$ is a C.I.

Corollary 2. If $A$ is Gorenstein ring (resp. is a C.I.) having a subfield $k$, and if $K$ is a finitely generated extension of $k$, then 
$A \bigotimes_{k} K$ is again Gorenstein (a C.I.).

Corollary 2'. If $f: X \rightarrow Y$ is a Gorenstein morphism (resp. is a morphism of C.I.) and if $Y^{\prime} \rightarrow Y$ is a morphism of finite type, so is $f_{\left(Y^{\prime}\right)}: X^{\prime}=X \times{ }_{Y} Y^{\prime} \rightarrow Y^{\prime}$.

Proof. By the induction on the number of generators of $K$ over $k$, we may assume $K=k(x)$. If $x$ is transcendental over $k$, then $A \otimes_{k} K$ is a localization of $A[X]$ and our assertion follows from Corollary 1. If $x$ is algebraic over $k$, then $K \cong k[X] / f(X))$ and $A \bigotimes_{k} K \cong A[X] / f(X) A[X], f(X)$ is not a zero divisor in $A[X]$ and again our assertion follows from Corollary 1.

Theorem 2. Let $B$ and $C$ be A-algebras, $B$ is flat over $A$ and $C$ is finitely generated over $A$. Then:

(1) If $A, B$ and $C$ are Gorenstein, then $B \otimes_{A} C$ is Gorenstein.

(2) If $B$ is a C.I. and $A$ and $C$ are regular, then $B \otimes_{A} C$ is a C.I.

Proof. By Theorem 1, the morphism $\operatorname{Spec}(B) \rightarrow \operatorname{Spec}(A)$ is Gorenstein. So, by Corollary $2^{\prime}, \operatorname{Spec}\left(B \otimes_{A} C\right) \rightarrow \operatorname{Spec}(C)$ is Gorenstein. And again by Theorem 1, the theorem follows. The same argument for the C.I. case.

Remarks. 1. The assertions of Theorem 1; " $A$ and $B / \mathfrak{m} B$ are Gorenstein $\Rightarrow B$ is Gorenstein" is in Hartshorne [3], and " $B$ is Gorenstein $\Rightarrow A$ is Gorenstein" is in Iversen [4]. The proof is different from ours.

2. In the notations of Theorem 1 , we should like to assert; " $B$ is a C.I. $\Leftrightarrow A$ and $B / \mathrm{m} B$ are C.I." By means of the characterization of C.I. in [5], the assertion is valid, for example, if we assume $\mathrm{n} B=\mathfrak{n}$. But we cannot determine the general case, yet.

\section{Part II}

In this part we shall prove the following

Theorem. Let $A$ and $B$ be two Gorenstein (resp. Macaulay) 
rings containing a common field $K$. Assume that $A \otimes_{K} B$ is noetherian and $A / \mathrm{m}$ is finitely generated over $K$ for each maximal ideal $\mathrm{m}$ of $A$. Then $A \otimes_{K} B$ is also a Gorenstein (resp. Macaulay) ring.

From now on, a "ring" always means a commutative noetherian ring with an identity element.

We begin with an easy

Lemma. Let $R$ be a 0 -dimensional local ring with the maximal ideal $\mathrm{m}$. Then the following conditions are equivalent:

(1) $R$ is a Gorenstein ring, i.e. 0 is irreducible.

(2) $0: \mathrm{n} \cong R / \mathrm{m}$.

(3) There exists a large*) principal ideal aR such that $(0: a)$ is irreducible.

Proof. (1) $\Rightarrow(2)$ : Let $x$ and $y$ be non-zero elements in $(0: \mathfrak{m})$. Then $x R \cap y R \neq 0$. If $r x=s y$ is a non-zero element in $x R \cap y R$, then $s$ and $r$ are units of $R$ and hence $x R=y R$. Therefore $(0: m)$ is simple.

$(2) \Rightarrow(3)$ : Obviously $(0: \mathrm{nt})$ is the required principal ideal.

$(3) \Rightarrow(1)$ : If 0 is reducible, then $0=x R \cap y R$ for some non-zero elements $x, y$ of $R$. Since $(0: a)=(x R: a) \cap(y R: a)$ and $(0: a)$ is irreducible, we may have $(0: a)=(x R: a)$. It follows easily that $a R \cap x R=0$. Thus $a R$ being large, $x R=0$ which is a contradiction.

Proposition 1. If $R$ is a Gorenstein (or Macaulay) ring, so is the polynomial ring $R\left[X_{1}, \cdots, X_{n}\right]$.

Proof. In the case of Macaulay rings, this is well known ([6]). Also for Gorenstein rings it seems to be known, but, having been able to find no reference, we shall sketch a proof which is a modification of one in Macaulay case. First we may assume $n=1$. Let 9 be a maximal ideal of $R[X]$. We may further assume that $R$ is a

*) An ideal $a$ of $R$ is called large if $R$ is an essential extension of $a$, that is $\mathfrak{a} \cap \mathfrak{b} \neq 0$ for each non-zero ideal $\mathfrak{b}$ of $R$. 
local Gorenstein ring with the maximal ideal $\mathfrak{m}=\mathfrak{M} \cap R$. The image of $M$ in $(R / \mathfrak{m})[X]$ is generated by an element $f$ modulo $\mathfrak{m}$ of $(R / \mathfrak{m})[X]$, where $f$ is an irreducible monic polynomial in $R[X]$ and all of its coefficients are units of $R$. Then we have $M=m R[X]$ $+f R[X]$. Let $\mathfrak{q}=\left(a_{1}, \cdots, a_{d}\right) R$ be an irreducible m-primary ideal generated by a system of parameter in $R$. Now we have to prove that $(\mathfrak{q} R[X]+f R[X])$ is irreducible. Changing $R$ into $R / \mathfrak{q}$, we may assume that $R$ is 0 -dimensional and $\mathfrak{q}=0$. Therefore it is sufficient to prove $R[X] / \mathfrak{M}=(f R[X]: \mathfrak{M}) / f R[X]$. By the above Lemma, $R / \mathfrak{m} \cong(0: \mathfrak{m})=a R$ and $(R / \mathfrak{m}[X] \cong a R[X]$ as $R[X]$-modules. Under this isomorphism, $f$ modulo $\mathfrak{m}$ corresponds to af. Hence $R[X] / \mathfrak{M}=(R / \mathfrak{m})[X] /(f$ modulo $\mathfrak{n})(R / \mathfrak{m})[X] \cong a R[X] /$ af $R[X]$ $=a R[X] /(a R[X] \cap f R[X])=(a R[X]+f R[X]) / f R[X]$. On the other hand, we have $a R[X]+f R[X]=(f R[X]: \mathfrak{M})$. For, let $h \in(f R[X]: \mathfrak{M})$ and $h=f g+r, \operatorname{deg} r<\operatorname{deg} f$. Then $m r \in f R[X]$ for each $m \in \mathfrak{n}$. Compairing the degrees, we have $m r=0$, and hence $r \in(0: \mathfrak{m}) R[X]=a R[X]$, that is $h \in a R[X]+f R[X]$. Therefore $(f R[X]: \mathfrak{M}) \subseteq a R[X]+f R[X]$. The opposite inclusion is obvious.

Proposition 2. Let $R$ be a Gorenstein (resp. Macaulay) ring containing a field $K$, and let $L$ be a finitely generated extension field over $K$. Then, $R \otimes_{K} L$ is also a Gorenstein (resp. Macaulay) ring.

Proof. By induction, we may assume that $L$ is a simple extension field over $K$. Say $L=K(\alpha)$. When $\alpha$ is transcendental over $K$, we have $R \otimes_{K} L \cong R[X]_{S}$, where $S$ is a multiplicative set in $R[X]$ consists of the non-zero elements of $K[X]$. When $\alpha$ is algebraic over $K$, we have $R \otimes_{K} L \cong R[X] / f R[X]$, where $f$ is a nonzero element in $K[X]$. Therefore the result follows immediately from Proposition 1.

Proof of the Theorem:

Let $M$ be a maximal ideal of $A \otimes_{K} B$. We must prove that $\left(A \otimes_{K} B\right)_{\mathfrak{M} \mathcal{M}}$ is a local Gorenstein (or Macaulay) ring. Without loss 
of generality, we may assume that $A$ and $B$ be local rings with the maximal ideals $\mathfrak{n}$ and $\mathfrak{n}$ respectively and $\mathfrak{M} \cap A=\mathfrak{n}, \mathfrak{M} \cap B=\mathfrak{n}$. Suppose $\operatorname{dim} A \geq 1$, and $a$ is non-zero divisor in $\mathfrak{m}$, then $a \otimes 1$ is a non-zero divisor in $\mathfrak{M}$ and $A \bigotimes_{K} B /(a \otimes 1)\left(A \bigotimes_{K} B\right) \cong(A / a A) \bigotimes_{K} B$. By induction, we may assume that $\operatorname{dim} A=0$. Similarly we may also assume that $\operatorname{dim} B=0$. Since $\operatorname{dim}\left(A \otimes_{K} B\right)=0$, we need nothing to say in the case of Macaulay rings. In the Gorenstein case, by Lemma $A / \mathfrak{m} \cong(0: \mathfrak{m})$ and $(0: \mathfrak{m})$ is large in $A$. Hence $\left(A / \mathfrak{m} \otimes_{K} B\right)_{\mathfrak{M} \mathfrak{l}}$ $=\left(A \otimes_{K} B\right)_{\mathfrak{M} \mathbb{N}} /\left(\mathrm{m} \otimes_{K} B\right)_{\mathfrak{M}}$ is isomorphic to a large principal ideal $\alpha\left(A \otimes_{K} B\right)_{\mathfrak{M M}}$ of $\left(A \otimes_{K} B\right)_{\mathfrak{M M}}$. On the other hand, $\left(A / \mathfrak{m} \otimes_{K} B\right)_{\mathfrak{M}}$ is a 0-dimensional Gorenstein local ring by Proposition 2, hence $((0: \alpha)$ in $\left.\left(A \otimes_{K} B\right)_{\mathfrak{M} \mathcal{R}}\right)=\left(\mathrm{m} \otimes_{K} B\right)_{\mathfrak{M} \mathcal{M}}$ is irreducible. Therefore again by Lemma, $\left(A \otimes_{K} B\right)_{\mathfrak{M}}$ is Gorenstein.

Remark: The assumption in Theorem that $A \otimes_{K} B$ is noetherian and $A / \mathfrak{m}$ is finitely generated over $K$ for each maximal ideal $\mathfrak{m}$ of $A$, is satisfied, for instance, when $A$ is a ring of finitely generated type over $K$, that is a quotient ring of a finitely generated ring over $K$.

\section{Part III}

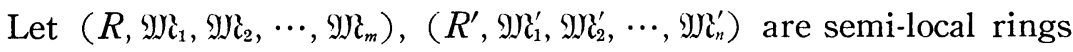
which are modules over a field $k$. Set $T=R \bigotimes_{k} R^{\prime}$. If one of $R / \mathfrak{M} i_{i}$ and $R^{\prime} / \mathfrak{M} \mathcal{M}_{j}^{\prime}$ is finitely generated over $k$, then $T /\left(\mathfrak{M}_{i} T+\mathfrak{M}_{j}^{\prime} T\right)$ is noetherian and every prime divisor $\mathfrak{p}$ of $\mathfrak{M M}_{i} T+\mathfrak{M}_{j}^{\prime} T$ is a minimal prime divisor. Then we see the following lemma.

Lemma 1. $T /\left(\mathfrak{M} \mathfrak{l}_{i} T+\mathfrak{M}_{j}^{\prime} T\right)=\left(R / \mathfrak{M} \mathfrak{N}_{i}\right) \bigotimes_{k}\left(R^{\prime} / \mathfrak{M} \mathfrak{l}_{j}^{\prime}\right)$

Now, we assume that $R, R^{\prime}$ are the Gorenstein rings and that $t_{1}, t_{2}, \cdots, t_{r}$ and $t_{1}^{\prime}, t_{2}^{\prime}, \cdots, t_{s}^{\prime}$ are the system of parameters, in the sense that $\sum t_{c} R$ and $\sum t_{e^{\prime}}^{\prime} R^{\prime}$ are the primary ideals belong to $\mathfrak{M}_{i}$ and $\mathfrak{M}_{j}^{\prime}$ respectively. Then $\bar{R}=R /\left(t_{1}, t_{2}, \cdots, t_{r}\right)$ and $\bar{R}^{\prime}=R^{\prime} /\left(t_{1}^{\prime}, t_{2}^{\prime}, \cdots, t_{s}^{\prime}\right)$ have only one minimal ideals $\mathfrak{T}_{i}$ and $\mathfrak{I}_{j}^{\prime}$ respectively. We denote the local tensor product of $R$ and $R^{\prime}$ by $R \times R^{\prime}$. 
Lemma 2. Notation as above. If $R^{\prime} / \mathfrak{M}_{j}^{\prime}$ is finitely generated over $k$, then every minimal ideal in $\bar{R} \times \overline{R^{\prime}}$ is contained in the ideal $\left(\mathfrak{N}_{i} \otimes \mathfrak{N}_{j}^{\prime}\right) \bar{R} \times \overline{R^{\prime}}$ generated by $\mathfrak{N}_{i} \otimes \mathfrak{N _ { j } ^ { \prime }}=\left\{\sum n \otimes n^{\prime} \mid n \in \mathfrak{N}_{i}, n^{\prime} \in \mathfrak{N}_{j}^{\prime}\right\}$.

Proof. We see that $\mathfrak{N}_{i} \otimes \mathfrak{Y}_{j}^{\prime} \subseteq \bar{R} \bigotimes_{k} \bar{R}^{\prime} \subseteq \bar{R} \times \bar{R}^{\prime}$, and see that $\mathfrak{N}_{i} \otimes \mathfrak{N}_{j}^{\prime}$ is an ideal in $\bar{R} \bigotimes_{k} \bar{R}^{\prime}$. Now we consider the ideal generally by $\mathfrak{N}_{i} \otimes \mathfrak{N}_{j}^{\prime}$ in $\bar{R} \times \bar{R}^{\prime}$ which is denoted by $\left(\mathfrak{N}_{i} \otimes \mathfrak{N}_{j}^{\prime}\right) \bar{R} \times \overline{R^{\prime}}$. We identify maximal ideals $\mathfrak{M}_{i}$ and $\mathfrak{M l}_{j}^{\prime}$ with subsets $\mathfrak{M l}_{i} \otimes 1$ and $1 \otimes \mathfrak{M}_{j}^{\prime}$ in $\bar{R} \otimes_{k} \overline{R^{\prime}}$, respectively. Let $c=\sum a_{p} \otimes b_{p}$ where $a_{p} \in \bar{R}$ and $b_{p} \in \bar{R}^{\prime}$. If $c\left(1 \otimes \mathfrak{M i _ { j } ^ { \prime }}\right)$ $=0$ and $c\left(\mathfrak{M}_{i} \otimes 1\right)=0$ then $c \in \mathfrak{N}_{i} \otimes \mathfrak{N}_{j}^{\prime}$. Because; $c\left(1 \otimes \mathfrak{M}_{j}^{\prime}\right)=0$ means $\left(\sum a_{p} \otimes b_{p}\right)\left(1 \otimes \mathfrak{M i}_{j}^{\prime}\right)=0$ namely $\sum a_{p} \otimes b_{p} m^{\prime}=0$ for all $m^{\prime} \in \mathfrak{M i}_{j}^{\prime}$. Now, let $a_{1}, a_{2}, \cdots, a_{u}$ be the linearly independent basis for $\sum a_{p} \bar{R}$, then we can see $a_{u+l}=\sum_{\beta \leq a} a_{\beta} A_{l \beta}\left(A_{l \beta} \in k\right)$. Therefore $\sum_{p} a_{p} \otimes b_{p}=a_{1} \otimes b_{1}+a_{2} \otimes b_{2}$ $+\cdots+a_{u} \otimes b_{u}+\sum_{l}\left(\sum_{\beta=1}^{u} a_{\beta} A_{l \beta} \otimes b_{u+l}\right)=\sum_{p=1}^{u} a_{p} \otimes b_{p}^{\prime}$, thus we may assume that all the $a_{p}$ are linearly independent over $k$. Since $\sum a_{p} \otimes b_{p} m^{\prime}=0$, and since $a_{p}(p=1,2, \cdots, u)$ are the linearly independent we see that $b_{p} m^{\prime}=0$ which means $b_{p} \in \mathfrak{N}_{j}^{\prime}$. Similarly we get $a_{p} \in \mathfrak{N}_{i}$ and therefore $c=\sum a_{p} \otimes b_{p} \in \mathfrak{N}_{i} \otimes \mathfrak{N}_{j}^{\prime}$. Thus, if we assume that the element $c$ of $\bar{R} \otimes \bar{R}^{\prime}$ is contained in a minimal ideal of $\bar{R} \otimes \overline{R^{\prime}}$, then $c \in \mathfrak{N}_{i} \otimes \mathfrak{N}_{j}^{\prime}$. $\bar{R} \otimes \bar{R}^{\prime} \rightarrow \bar{R} \times \bar{R}^{\prime}$ is an injection and since $\bar{R} \times \bar{R}$ is a ring of quotient of $\bar{R} \otimes \bar{R}^{\prime}$, every minimal ideal of $\bar{R} \times \bar{R}^{\prime}$, lies over a minimal ideal of $\bar{R} \otimes \bar{R}^{\prime}$ and we have the result.

Lemma 3. With the same notation as above, $\left(\mathfrak{N}_{i} \otimes \mathfrak{N}_{j}^{\prime}\right) \bar{R} \times \overline{R^{\prime}}$ $\cong\left(R / \mathfrak{M C}_{i}\right) \times\left(R^{\prime} / \mathfrak{M l}_{j}^{\prime}\right)$ as $\bar{R} \times \bar{R}^{\prime}$-modules.

Proof. Let $a$ be a non-zero element in $\mathfrak{l}_{i}$. Then $0 \neq a \bar{R} \subseteq \mathfrak{N}_{i}$. $\mathfrak{N}_{i}$ is a minimal ideal in $\bar{R}$ and therefore $a R=\mathfrak{U}_{i}$. On the other hand $a \mathfrak{M}_{i}=0$ and therefore $\mathfrak{Y} \mathfrak{l}_{i} \cong R / \mathfrak{M}_{i}$. Similarly we get $\mathfrak{\mathfrak { U } _ { j } ^ { \prime }} \cong R^{\prime} / \mathfrak{M}_{j}^{\prime}$, therefore $\left(\mathfrak{I}_{i} \otimes \mathfrak{I}_{j}^{\prime}\right) \bar{R} \times \overline{R^{\prime}} \cong\left(R / \mathfrak{M}_{i}\right) \times\left(R^{\prime} / \mathfrak{M i}_{j}^{\prime}\right)$ as $\bar{R} \times \overline{R^{\prime}}$-modules.

(q.e.d.)

Now we see that $\bar{R} \times \bar{R}^{\prime}=L_{1} \oplus L_{2} \oplus \cdots \oplus L_{d}$, where $L_{\alpha}$ are the local rings and $d$ is the number of prime divisors of zero. 
Lemma 4. Let $\mathfrak{M}$ be one of maximal ideals in $\bar{R} \times \overline{R^{\prime}}$ and $\mathfrak{p}_{\alpha}$ the maximal ideal of $L_{\alpha}$. Then we can see that there is a suitable $\beta$ such that $\left(\bar{R} \times \bar{R}^{\prime}\right)_{\mathfrak{M}}=\left(L_{\beta}\right)_{\mathfrak{p}_{\beta}}$.

Proof. We can assume that $\mathfrak{M}=L_{1} \oplus L_{2} \oplus \cdots \oplus L_{\beta-1} \oplus \mathfrak{p}_{\beta} \oplus L_{\beta+1} \oplus$ $\cdots \oplus L_{d}$, therefore $\left(\bar{R} \times \overline{R^{\prime}}\right)_{\mathfrak{M}}=\left(L_{\beta}\right)_{\mathfrak{p}_{\beta}}$ for suitable $\beta$.

Lemma 5. Let $\left(\mathfrak{N}_{i} \otimes \mathfrak{N}_{j}^{\prime}\right) \bar{R} \times \bar{R}^{\prime}=N_{1} \oplus N_{2} \oplus \cdots \oplus N_{d}$, then each $N_{\alpha}$ contains a unique minimal ideal in $L_{\alpha}$, and conversely, a minimal ideal in $\bar{R} \times \bar{R}^{\prime}$ is contained in one of $N_{\beta}$ for suitable $\beta$.

Proof. By Lemma 3, we see that $\left(\mathfrak{N}_{i} \otimes \mathfrak{N}_{j}^{\prime}\right) \bar{R} \times \bar{R}^{\prime} \cong\left(R / \mathfrak{M}_{i}\right)$ $\times\left(R^{\prime} / \mathfrak{M}_{j}^{\prime}\right)$ as $\bar{R} \times \bar{R}^{\prime}$-modules. Since $R / \mathfrak{M}_{i}$ and $R^{\prime} / \mathfrak{M}_{j}^{\prime}$ are fields $\left(R^{\prime} / \mathfrak{M}_{j}^{\prime}\right.$ being finitely generated), we see that $\left(R / \mathfrak{M}_{i}\right) \times\left(R^{\prime} / \mathfrak{M}_{j}^{\prime}\right)$ is Gorenstein. Therefore each $N_{\alpha}$ contains a unique ideal in $L_{\alpha}$. A minimal ideal in $\bar{R} \times \bar{R}^{\prime}$ is contained in $\left(\mathfrak{N}_{i} \otimes \mathfrak{N}_{j}^{\prime}\right) \bar{R} \times \bar{R}^{\prime}$, hence contained in some $N_{\beta}$.

Let $\mathfrak{\Im}$ be the Jacobson radical of $R \times R^{\prime}$ and set $\Re=\bigcap_{n} \mathfrak{\Im}^{n}$. Then complete tensor product $R \bar{\otimes} R^{\prime}=$ (the completion of $R \times R^{\prime} / \Omega$ ) is a semi-local ring, because every maximal ideal of $R \bar{\otimes} R^{\prime}$ has a finite basis.

Thus, we can get the next theorem.

Theorem 6. $R \bar{\otimes} R^{\prime}$ is Gorenstein ring, provided that $R^{\prime} / \mathfrak{M} \mathcal{M}_{j}^{\prime}$ is finitely generated for every $j$.

Corollary 7. Under the same assumptions as above, let $R^{\prime \prime}$ be the semi-local ring which is a dense subspace of $R \otimes R^{\prime}$, then $R^{\prime \prime}$ is Gorenstein. 


\section{References}

[1] H. Bass: On the ubiquity of Gorenstein rings, Math. z. 82 (1963).

[2] A. Grothendieck: Éléments de Géométrie Algébrique.

[3] R. Hartshorne: Residues and Duality, Springer (1966).

[4] B. Iversen: On flat extensions of Noetherian rings, Proc. Amer. Math. Soc. 16 (1965).

[5] G. Scheja: Über die Bettizahlen lokaler Ringe, Math. Ann. 155 (1964).

[6] M. Nagata: Local rings. (Interscience Tracts, 1962).

[7] O. Zariski and P. Samuel: Commutative Algebra, Vol. 2. Van Nostrand. Princeton (1960).

Tokyo University

Tokyo Metropolitan University

Kyoto University 